\title{
Old maids have more appeal: Effects of age and pheromone source on mate attraction in an orb-web spider
}

Anna-Lena Cory, Jutta M Schneider

Background. In many insects and spider species, females attract males with volatile sex pheromones, but we know surprisingly little about the costs and benefits of female pheromone emission. Here we test the hypothesis that mate attraction by females is dynamic and strategic in the sense that investment in mate attraction is matched to the needs of the female. We use the orb-web spider Argiope bruennichi in which females risk the production of unfertilised egg-clutches if they do not receive a copulation within a certain time-frame. Methods. We designed field experiments to compare mate attraction by recently matured (young) females with females close to oviposition (old). In addition, we experimentally separated the potential sources of pheromone transmission, namely the female body and the web silk. Results. In accordance with the hypothesis of strategic pheromone production, the probability of mate attraction and the number of males attracted differed between age classes. While the bodies and webs of young females were hardly found by males, the majority of old females attracted up to two males within two hours. Old females not only increased pheromone emission from their bodies but also from their webs. Capture webs alone, spun by old females were significantly more efficient in attracting males than webs of younger females. Discussion. Our results suggest that females modulate their investment in signalling according to the risk of remaining unmated and that they thereby economize on the costs associated with pheromone production and emission. 
1 Old maids have more appeal: Effects of age and pheromone source on mate attraction in

2 an orb-web spider

3 Anna-Lena Cory ${ }^{1}$, Jutta M. Schneider ${ }^{1}$

4 'Zoologisches Institut, Universität Hamburg, Hamburg, Hamburg, Germany

5

6 Corresponding author:

7 Anna-Lena Cory ${ }^{1}$

8 Martin-Luther-King-Platz 3, Hamburg, 20146, Germany

9 Email address: anna lena.cory@yahoo.de

10 


\section{Abstract}

13 Background. In many insects and spider species, females attract males with volatile sex pheromones, but we know surprisingly little about the costs and benefits of female pheromone emission. Here we test the hypothesis that mate attraction by females is dynamic and strategic in the sense that investment in mate attraction is matched to the needs of the female. We use the orb-web spider Argiope bruennichi in which females risk the production of unfertilised eggclutches if they do not receive a copulation within a certain time-frame.

Methods. We designed field experiments to compare mate attraction by recently matured (young) females with females close to oviposition (old). In addition, we experimentally separated the potential sources of pheromone transmission, namely the female body and the web silk. Results. In accordance with the hypothesis of strategic pheromone production, the probability of mate attraction and the number of males attracted differed between age classes. While the bodies and webs of young females were hardly found by males, the majority of old females attracted up to two males within two hours. Old females not only increased pheromone emission from their bodies but also from their webs. Capture webs alone, spun by old females were significantly more efficient in attracting males than webs of younger females.

Discussion. Our results suggest that females modulate their investment in signalling according to the risk of remaining unmated and that they thereby economize on the costs associated with pheromone production and emission. 


\section{Introduction}

Mating partners have to meet in order to mate and reproduce. This prerequisite may be a trivial aspect in group-living animals, but it can constitute a significant factor within the overall costs of sexual reproduction in other taxa (Kokko \& Wong 2007; Magnhagen 1991). Encounters with sexual partners can be facilitated by a wide range of mechanisms, e.g. meeting at certain locations (Hoglund \& Alatalo 1995; van Ginneken \& Maes 2005) or producing long-range signals that attract potential mates (Gerhardt 1994; Roelofs 1995). The latter can occur via different communication channels (visual, acoustic or chemical), but which modality evolved will depend on the distance the signal has to travel in addition to other biotic and strategic selection pressures on signal design (Endler 1993). Here, we are concerned with chemical communication that is considered to be an evolutionary ancient modality demanding the lowest cost in comparison to acoustic and visual signals (Bradbury \& Vehrencamp 1998). However, this assumption is largely untested.

Both males and females can produce sex pheromones to attract mates (Tillman et al. 1999; Wyatt 2014), but particularly mate attraction by females is poorly understood. In many moths and spiders, females produce volatile or substrate bound pheromones, and males follow these traces (Gaskett 2007; Greenfield 1981; Wall \& Perry 1987). Selection on male signal perception has led to astounding sensory sensibility and accuracy, as exemplified by the receptor density and functionality on the antennae of the male moth, Bombyx mori (Kaissling \& Priesner 1970;

Steinbrecht 1970). The solid understanding of natural selection on males as signal recipients is opposed by a rudimentary comprehension of the female perspective. It is largely unstudied but nevertheless debated, whether pheromones emitted for mate attraction by females can be classified as sexual signals that actively transmit information to potential mates or as cues 
56 exploited by males (Andersson 1994; Harari \& Steinitz 2013; Williams 1992; Zuk \& Kolluru

57 1998). We use the orb-web spider Argiope bruennichi Scopoli, 1772 to test predictions from the

58 hypothesis that pheromones are costly sexual signals.

59 Umbers, Symonds and Kokko (2015) recognized that the emission of cues that reveal one's

60 presence and location will not only reach the intended receiver, but likely attracts parasitoids and

61 predators in addition to mates (e. g. Arakaki et al. 1996; Noldus et al. 1991; Zegelman et al.

62 1993). Besides increased mortality, the attraction of an adversely high number of mates also

63 involves costs (Arnqvist \& Nilsson 2000). In spiders, the presence of males may be costly

64 because of kleptoparasitism (Erez, Schneider, \& Lubin, 2005), web damage (Anava \& Lubin, 1993; Harari, Ziv, \& Lubin, 2009; Watson, 1986), mate guarding (Calbacho-Rosa, CordobaAguilar, \& Peretti, 2010; Fromhage \& Schneider, 2005; Herberstein et al., 2005), and sexual harassment (Foellmer \& Fairbairn 2003; Lubin 1986; Robinson \& Robinson 1973). Hence, even if the physiological costs of pheromone production would be low, extrinsic costs could be strong enough to select for a strategic use of pheromones.

In many spider species, including A. bruennichi, females are under pressure to attract males soon after maturation because egg maturation advances regardless of whether or not females have copulated. Hence, females that have not been found by a male in time will produce unfertilised egg clutches (Welke \& Schneider 2012). Therefore, if virgin females use pheromones strategically they should increase signalling efforts with increasing number of days passed since maturation (here called age).

Following this line of reasoning, apart from their location, female spiders can be expected to

77 inform males about receptivity, mating status and perhaps even quality by regulating attraction signals. There is ample evidence that male spiders distinguish between virgin and mated females 
79 (Schulte et al. 2010; Stoltz et al. 2007; Tuni \& Berger-Tal 2012; Watson 1986). Indeed, in many

80 species females seem to loose receptivity after mating (Herberstein et al. 2002; Rabaneda-Bueno

81 et al. 2008), but they may regain receptivity when sperm supplies require replenishing

82 (Perampaladas et al. 2008). Some studies have shown that virgin females produce sex

83 pheromones that are absent in mated females (Chinta et al. 2010; Jerhot et al. 2010; Schulz \&

84 Toft 1993). However, it is unknown whether the emission of sex pheromones by mated females

85 discontinues because females are in control or because males avoid sperm competition by

86 transferring pheromonostatic compounds (Arnqvist \& Rowe 2005; Thomas 2011).

87 Note that in spiders, pheromones for long-range attraction are volatile but seem not to be

88 released by a specific gland, rather they are found in the body and the silk (Gaskett 2007; Schulz

89 2013; Schulz et al. 2004). Many studies demonstrated that chemical compounds on female spider

90 silk contain important information for males (Johnson et al. 2011; Stoltz et al. 2007; Sweger et

91 al. 2010; Tuni \& Berger-Tal 2012). Gaskett (2007) speculated that silk-based pheromones are

92 less costly while the emission of body pheromones might be more adjustable. Beyond

93 speculations, no studies on web-building spiders compared if female bodies and web silk differ

94 in the efficiency of mate attraction.

95 Here, we investigated age-dependency of male attraction by pheromones associated with the web

96 or the female body. We conducted a field experiment with the European wasp spider $A$.

97 bruennichi, in which the sex pheromone is known and found on the body and web of virgin

98 females (Chinta et al. 2010). Using the synthesized pheromone, Chinta et al. (2010) also found

99 that male attraction was dose-dependent. The presence of a response to pheromone concentration

100 might imply that variation occurs in nature and that females vary in their pheromone emission.

101 We predict that the longer females remain unmated, the more they should increase their 
102 investment in sex pheromone emission to attract males from larger distances and to outcompete 103 other females in the proximity. If female signalling is costly for A. bruennichi females, we would 104 expect that old females regularly attract more males than necessary but accept the costs because 105 laying unfertilized egg clutches is even more costly. Note that only one mating event is needed to 106 fertilize all eggs (Schneider et al. 2005) and that due to efficient mate plugging by males, females

107 are limited to use sperm of two males at most (Nessler et al. 2007). Field experiments are ideal 108 for testing this prediction since they not only allow the qualitative assessment of wide-range 109 attraction but also provide information about the intensity of mate attraction by counting the 110 number of male arrivals. Females would benefit from regulating the emission within a short time

111 frame because the mating season only lasts for 3-4 weeks (Zimmer et al. 2012). Strategic

112 signalling implies that with increasing age and pressure to attract males, unmated females should 113 emit more pheromones.

114 Following this, we hypothesise that (1) mate attraction by virgin females increases with post115 maturation age. To further understand how females enhance mate attraction, we tested females 116 and their webs separately. Modulating pheromone content of webs requires rebuilding of webs

117 and is, hence, less rapid than direct emission from the body. Thus, we hypothesise that (2)

118 females adjust pheromone production on their web later than on the female body when the need 119 of signalling is strikingly increased.

121 Material \& Methods

122 Collection and Maintenance

123 We collected 59 juvenile and sub-adult Argiope bruennichi females on a natural meadow near

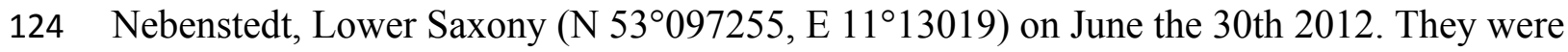


125 housed under laboratory conditions at ambient temperature and natural photoperiod and were

126 sprayed with water five days a week. Depending on their body size, females were kept in $250 \mathrm{ml}$

127 or $500 \mathrm{ml}$ cups that had a hole stuffed with cotton wool on the top. Sub-adult females were

128 transferred into Perspex frames $(35 \times 35 \times 6 \mathrm{~cm})$ where they build a normal orb-web. Small

129 juveniles were fed with approximately 20 Drosophila hydei two times a week. Large juvenile,

130 sub-adult and adult females received three Calliphora two times a week (for exception see

131 below).

132 Female body measurements

133 Sub-adult females were inspected for moults on at least five days a week. Moults to maturity

134 were recognized by inspection of the genitalia. The external genitalia of adult females have a 135 spoon-shaped structure, the scape. In the sub-adult stage, the scape starts to develop but is 136 covered with a thin-skinned layer. Mature females lose this layer, and the scape is exposed. At

137 maturity, females were allocated in a pre-set order to the three age treatments. On the first day 138 after maturation females were weighed on a calibrated scale (Mettler Toledo AB54-S) with an 139 accuracy of $0.1 \mathrm{mg}$. Each female was also weighed on the day of the test (test weight).

140 Spider size was determined after the experiments. Females were brought back to the laboratory 141 where they were kept and well supplied with food and water until natural death occurred. After 142 death, the first pair of legs was removed and photographed under a stereomicroscope. We 143 measured the length of the two segments tibia and patella with the Leica IM500 Imaging 144 software (version 4.0; Leica Microsystems Imaging Solution Ltd., Cambridge, UK). We used 145 this length as an approximation for the overall spider size. Legs and other sclerotized body parts 146 do only change through moulting but not between moults. As an approximation of female 
147 condition, we used the residuals of the regression of body weight at maturation or at the test day

148 and size of the females (Schulte-Hostedde et al. 2005). To achieve a normal distribution, we log-

149 transformed the data for test weight.

\section{Pheromone source}

151 To disentangle the female body or the web as the source of pheromones, we used the webs of

152 sub-adult females as "neutral webs" because they do not contain pheromones (Chinta et al.

153 2010). In the "female body" trials, virgin females were placed into these neutral webs. Most

154 females showed little activity on the web and seemed to accept it. However, bad weather

155 conditions led to more activity such that some females either stabilized the web with silk threads

156 or destroyed the neutral web (see "Data analysis"). For the "web" trials, freshly constructed webs

157 by virgins were used without the female. Generally, the web and its producer female were used

158 on the same day (exceptions arise when webs are destroyed before they can be placed in the

159 meadow). We ensured that the web was freshly built on the day of the trial by destroying webs

160 on the evening before the trial. Females built their webs in the early morning, and we had

161 introduced a Calliphora fly into the frame to stimulate the production of a capture web.

\section{Female age classes}

163 Oviposition can already occur 10-16 days after maturation. We used this time window to define

164 three age classes of virgin females. Age is given as the number of days that had passed since

165 maturation. "Young females" were 1-2 days adult, "middle-aged females" 3-7 days adult (5.8 \pm

166 1.4), and "old females" had matured 8 or more days ago $(10.0 \pm 2.1)$. The webs were produced

167 by females that were 1-2 days old in the groups of young females, by middle-aged females of 5.0

$168 \pm 1.3$ days and by old females of the average age of $10.0 \pm 2.8$ days. We used a 2-day time 
169 window for the category of young females because binary choice experiments in the laboratory

170 have shown that males do not distinguish between sub-adult females and females younger than

171 three days (Schneider et al. 2016). A plausible explanation for this result is that females do not

172 produce the pheromone until the 3rd day after maturation. Nevertheless, females readily copulate

173 with males on the day of sexual maturity (Welke et al. 2012). An age of at least eight days was

174 used for the category "old females" to include females with high pressure to mate because

175 oviposition is very close and the risk of producing unfertilised eggs is very high. Each female

176 was used once for only a single age class.

177 A comparison of mate attraction by females of different ages requires testing a matched sample

178 of females from all age classes every day. This is important since conditions on subsequent days

179 are rarely the same: males disappear from the mating pool as they get cannibalised and male

180 activity depends on weather conditions. However, females mature very synchronously which

181 makes it difficult to find young virgins towards the end of the mating season (Zimmer et al.

182 2012). To achieve a balanced availability of young and old females during the test period, we

183 included a short period of food restriction during the final instars for half of the females which

184 received three Calliphora flies once instead of twice a week for about two weeks. Spiders are

185 adapted to endure periods of prey shortage since they regularly occur in nature. A short hunger

186 period does not negatively affect spider survival or fecundity. However, it is sufficient to

187 postpone moulting into maturity for a few days. We payed attention to feed all females well

188 shortly before adulthood to standardize hunger level and distributed females from the two

189 feeding regimes across all three age classes to avoid a systematic bias.

190 To account for the possibility that differences in juvenile feeding history might have an impact

191 on the condition at maturation (Hector \& Nakagawa 2012) and influenced our age treatments, we 
192 tested whether the body condition on the first day of sexual maturity differed between females of 193 different age classes. We found no significant differences (Table 1) but in the web trial, there 194 was a trend that females allocated to the "middle-aged" group initially had a higher condition 195 than the others, while young females had a lower condition. However, this would be only 196 problematic if we found that middle-aged females attracted the most males.

197 Naturally, body condition increases with age (ANOVA on the weight of females across the age 198 classes: $F_{2,26}=21.44, p<0.0001$; ANOVA on the weight of females that produced the webs: $\left.199 F_{2,29}=21.32, p<0.0001\right)$ such as body weight does. This increase is due to feeding and egg200 maturation. Hence, age and condition are confounding factors and cannot be separated. In an 201 attempt to dissociate the effects of female age and condition, we tested the effect of female 202 condition on male attraction separately within each age class. 
212 Table 1: Female weight and female size information within the age classes and statistical

213 comparisons (ANOVA) of female weight and female size between the age classes

Adult weight $(\mathrm{mg})^{1} \quad$ Size $(\mathrm{mm})^{2} \quad$ Condition $^{3}$

$\mathrm{N} \quad \mathrm{Mean} \pm \mathrm{SD} \quad \mathrm{N} \quad \mathrm{Mean} \pm \mathrm{SD} \quad \mathrm{N} \quad$ Mean $\pm \mathrm{SD}$

Female body trials

\begin{tabular}{lcrcccc}
\hline Young & 12 & $92.2 \pm 25.5$ & 10 & $6.2 \pm 0.6$ & 10 & $-5.6 \pm 5.9$ \\
Middle-aged & 12 & $94.0 \pm 25.3$ & 10 & $6.1 \pm 0.6$ & 10 & $4.5 \pm 17.3$ \\
Old & 12 & $83.4 \pm 25.3$ & 10 & $6.0 \pm 0.6$ & 10 & $1.5 \pm 7.1$ \\
\hline ANOVA & $F_{2,33}=0.68, p=0.5148$ & $F_{2,26}=0.35, p=0.7053$ & $F_{2,26}=2.10, p=0.1425$ \\
\hline
\end{tabular}

Web trials

\begin{tabular}{lcrlclc}
\hline Young & 14 & $96.0 \pm 27.5$ & 11 & $6.2 \pm 0.6$ & 11 & $-6.6 \pm 6.3$ \\
Middle-aged & 14 & $98.2 \pm 27.9$ & 11 & $6.2 \pm 0.6$ & 11 & $4.4 \pm 16.4$ \\
Old & 13 & $80.1 \pm 23.2$ & 10 & $5.9 \pm 0.6$ & 10 & $2.9 \pm 11.4$ \\
\hline ANOVA & $F_{2,38}=1.88, p=0.1664$ & $F_{2,29}=1.22, p=0.3105$ & $F_{2,29}=2.88, p=0.0725$
\end{tabular}

$214{ }^{1}$ The adult weight was measured shortly after maturity. ${ }^{2}$ For the size, we used the tibia-patella

215 length (segments of the leg) as a good approximation for the general size of spiders. ${ }^{3}$ Residuals

216 of the regression of body weight shortly after maturation and size of the females.

218 Test preparation (field)

219 Field experiments were conducted between $12^{\text {th }}$ and $26^{\text {th }}$ July 2012 on a natural meadow in

220 Buxtehude, Lower Saxony (N 53453995, E 9674402). Our experimental period covered most 
221 of the mating season, although some males were still active when the trials ended. We observed

222 male activity a few days before the experiments started and picked the experimental areas

223 according to similar male activity.

224 The design was balanced in that we tested females of all age classes on each test day. Females

225 were transported in containers (volume of $120 \mathrm{ml}$ ) to the field site and the webs for transported

226 within the frames in specially designed transport boxes to minimise damage. On the test

227 meadow, the Perspex frames with webs of the study females ("web" trial) or non-pheromone

228 webs ("female body" trial) were fixed with tent pegs to the ground in an upright position. To

229 ensure that the web was not destroyed by tall grasses, a small area around the frame was

230 flattened. The distribution of the frames on the meadow was random although we ensured that

231 the distance between the frames and local adult females was larger than $50 \mathrm{~cm}$. For the "female

232 body" trial, we carefully placed the females into the prepared neutral webs after the frames were

233 securely fixed on the ground.

234 Test run

235 "Female body" trials and "web" trials were conducted apart from each other to avoid pheromone

236 contamination. Either the two web categories had a distance of at least eight metres, or they were

237 put out at different times of the day. The order and position was reversed every day so that each

238 category was tested at each time of the day and in different parts of the meadow. The distance of

239 eight metres was dictated by the size and structure of the test meadow.

240 We observed each web for two hours and counted the number of male visitors. Since not all

241 males found their way into the web, we also counted males that apparently attempted to reach the

242 females or their webs for at least 5 minutes. An attempt was only counted when the male 
243 wandered around grass lying in close vicinity to the female. To avoid pseudo-replication, we

244 collected male visitors for the duration of the test. The males were released a few metres away

245 from the test area after the test run was ended. There was a half-hour break between subsequent

246 test runs so that the males could acclimatize in the field.

247 Data analysis

248 Although we were especially interested in the effects of female age and pheromone source, we

249 also analysed the effects of size, test weight, and condition. However, female weight and

250 condition are correlated and produced very similar results. We therefore excluded female weight

251 from the analysis.

252 Most likely due to windy and humid weather conditions, we had a high proportion of zero

253 counts. We divided the analysis into two levels and firstly used a binary response variable

254 namely whether or not males approached. Secondly, we explored the treatment effects on the

255 number of male visitors and reduced the sample to only include females that had male visitors.

256 The design was originally balanced with each 42 webs and females, but some webs and neutral

257 webs were destroyed before the tests could start. Overall, we could test 41 webs and 39 females

258 on neutral webs. However, particularly on rainy days some females escaped from the neutral

259 webs mostly and observation time was less than 120 minutes. Since, we found that most first

260 male visitors $(92 \%)$ appeared within the first hour of the test run, we discarded only 3 cases that

261 were shorter than 60 minutes and retained 36 tests that lasted for at least one hour for the first

262 analysis of male appearance as a binary variable. However, for the test of treatment effects on the

263 number of male visitors, we considered only females and their webs that were observed for the

264 full two hours and in which males visited, at least, the web or the female $(n=14)$. 
265 Statistical analyses were performed with the program $\mathrm{R}$ version 3.0.3. All variables were tested

266 for normal distribution with the Shapiro-Wilk test. We used parametric tests for normally

267 distributed data and nonparametric tests for analyses of non-normal and paired data. All tests

268 were two-tailed. Since only a part of the "female" and "web" data was paired, we performed a

269 generalised estimating equation (GEE) to explore whether females or webs were more likely to

270 attract males. We created a binary model with pheromone source as testing variable and defined

271 female ID as a random term (grouping variable). The correlation structure was specified as

272 “independent". We simplified the model by excluding the variable "pheromone source" and

273 compared whether the original model significantly differed from the simplified model by using

274 the Wald test. A significant difference between both models would be indicative for a significant

275 effect of the pheromone source on the probability of being visited by a male.

\section{Results}

278 Only 23 of 77 females or webs were visited by males during the 2 h-observation period, most of them (22 of 23 ) within the first 60 minutes. Presumably, male activity was low due to very humid and windy weather conditions. We included all trials into the analyses unless stated otherwise.

Females on neutral webs attracted visitors with a significantly higher probability (14 of 36 females) than webs alone ( 8 of 41 webs) (GEE: $X^{2}=6.44, N=77, p=0.0111$; Fig. 1$)$. A paired comparison of the number of male visitors attracted by a given female in a neutral web and by her own web was not significant (Wilcoxon signed rank test: $T=58.5, N=14, p=0.3724$ ) suggesting that pheromone emission from the body directly or via the silk of the web were not 
287 necessarily linked. However, the sample size was low as we only considered 14 pairs in which

288 males visited, at least, the web or the female.

289 Female body

290 We used Fisher exact tests for pairwise comparisons of the proportion of females on neutral

291 webs visited per age class. Although the probability of male visits increased with increasing age

292 class (Fig. 1), we only found a significant difference between young and old females $(N=24$,

$293 p=0.03607)$. Female fixed size (t-test: $\left.t_{27}=-0.67, p=0.5079\right)$ did not influence the probability of a

294 male visit while female condition had significant positive effects (t-test: $t_{27}=-3.49, p=0.0017$, Fig.

295 2). Note that any potential effect of female body condition was confounded by age (see

296 methods). In an attempt to separate age and condition, we tested the effects of female condition

297 within the three age classes and found no significant differences in condition of females that

298 were or were not visited (young females: no value; middle-aged females: t-test: $t_{7}=-0.32$,

$299 p=0.7554$, old females: logistic regression: $X^{2}=0.99, N=10, p=0.3187$ ).

300 Excluding females that were never visited, females received 1 to 4 male visits (median=2) within

301 two hours. Due to the unbalanced sample sizes within the age classes (young females were

302 hardly visited at all), we dropped the analysis with the category "age class". Instead, we used

303 "post-mature age" as a continuous variable, but we did not find a significant correlation between

304 age and the number of male visitors (Spearman rank correlations: $r_{s}=0.49, N=13, p=0.0909$, Fig.

305 3). Interestingly, while visitation rates did not correlate with female size (Spearman rank

306 correlations: $\left.r_{s}=0.82, N=13, p=0.789\right)$, the number of male visitors significantly increased with

307 female condition (Spearman rank correlations: $r_{s}=0.68, N=13, p=0.0103$ ).

308 Web silk 
309 To compare the attractivity of webs without females, we used, as above, Fisher exact tests for

310 pairwise comparisons of the probability of being visited between female age classes. Since the

31114 webs of middle-aged females and the 14 webs of young females hardly attracted males at all

312 (only a single web was visited in each of the two groups), we compared each of both categories

313 only with the category "old females". The tests revealed that webs of old females (6 of 13 webs)

314 attracted males significantly more often than webs of young females and webs of middle-aged

315 females (for both pairwise comparisons we used the same statistic: $N=27, p=0.0329$, Fig. 1 ).

316 While size (t-test: $t_{30}=0.81, p=0.4224$ ) of the females that had produced the webs did not

317 significantly differ between visited and non-visited webs, the effect of condition was significant

318 (t-test: $t_{30}=-2.81, p=0.0086$; Fig. 2 ) suggesting that females in better condition produced webs

319 that were more attractive to males. However, remember that females in better condition were

320 significantly older (Spearman rank correlations: $r_{s}=0.74, N=32, p<0.0001$ ) and 8 of 10 webs

321 came from old females.

322 Most female webs were visited by one or two males ( $80 \%)$, although the maximum was five.

323 Visitation rates cannot be compared between the age classes because $80 \%$ of the visited female

324 webs were from old females, as already mentioned above. As with the female body, we

325 conducted the analysis with the continuous variable "post-mature age". The post-mature age

326 correlated positively with the number of males that approached the web (Spearman rank

327 correlations: $\left.r_{s}=0.69, N=10, p=0.0271\right)$. From a post-mature age of 14 days onwards, some

328 females were visited by more than two males (Fig. 3). Neither female size (Spearman rank

329 correlations: $\left.r_{s}=0.09, N=10, p=0.8149\right)$ nor female condition (Spearman rank correlations:

$\left.330 r_{s}=0.61, N=10, p=0.0613\right)$ were significantly related to the number of male visitors. 
332 Discussion

333 Our field assays provided support for the hypothesis of strategic pheromone emission. In

334 accordance with our prediction, females close to oviposition (old and heavy) were most

335 successful in mate attraction. Females used both, their body and their web to transmit

336 pheromones, although the latter became more relevant with increasing age of the female. As

337 oviposition came close, the attractivity of the web for mate attraction increased. Female fixed

338 size estimated from sclerotized body parts seemed to be irrelevant for male attraction while it

339 was indeed the body condition that caused increased numbers of males to visit a female and her

340 web. However, because of the correlative nature between female age and female condition, it is

341 difficult to separate both factors.

342 Our results are in accordance with model predictions by Umbers and colleagues (2015) in that

343 females increase their investment in signalling if they age without having encountered a mate

344 yet. Young virgin A. bruennichi females, particularly at the beginning of the mating season and

345 under high densities (Zimmer et al. 2012), are likely found haphazardly by the protandrous

346 roving males and females may save the costs of pheromone production. This is suggested by the

347 high frequency of mate guarding of sub-adult females in the field (Uhl et al. 2015). As females

348 age, egg maturation progresses and by mating too late, oviposition will take place, albeit laying

349 unfertilised eggs. Females should be under selection to avoid this fate. Thus, it is adaptive to

350 increase signalling effort if no males have appeared within a few days after maturation. Such

351 females may find themselves in a low-density patch or too late in the season when most of the

352 males are already mated and consumed (Zimmer et al. 2012).

353 Extrinsic costs of signalling can result from the attraction of parasitoids or an detrimentally high

354 number of mates. Male presence on the web is known to have costs for female spiders because 
355 prey capture is reduced, and predation risk is increased (Herberstein et al. 2002). In nature, $A$.

356 bruennichi females mate with one or two males (Zimmer et al. 2012) and a single copulation is

357 sufficient to fertilise all eggs (Schneider et al. 2005). During copulation males plug the paired

358 genital openings of females to prevent sperm competition. The mating plugs are highly effective

359 so that the majority of females can store and use sperm of two males at most (Nessler et al.

360 2007). In our study, old females attracted many more males than needed for fertilisation and

361 more than of the maximal number of sires. Furthermore, webs were especially used for mate

362 attraction when oviposition was very close. In fact, those webs attracted up to five males. The

363 larger surface of the web may increase pheromone distribution. Hence, females may use

364 pheromones strategically and remain hidden for males if they do not benefit from mate

365 attraction. We did not test whether old females build larger webs compared to young females.

366 Therefore, further studies should consider that females might increase pheromone distribution by

367 extending the web surface or by adding more pheromones onto the web.

368 While extrinsic costs are most likely present, it is unclear whether pheromone production in

369 these spiders has physiological costs as well. If signal production involves physiological costs,

370 females in better condition may emit more and would honestly signal their quality. A purely

371 strategic use does not require honesty because it would only imply increased signalling with

372 increasing age and pressure to attract males. However, it is difficult to separate female condition

373 from female age because females become fat as egg maturation proceeds. Accordingly, both

374 variables reflect the time pressure on females to secure fertilisation by increasing investment into

375 signalling. A more important influence of age is confirmed by binary choice tests in $A$.

376 bruennichi, in which males showed no significant preference when presented with a choice

377 between two young females of the same age that differed in condition and size (Schulte et al. 
378 2010). Investment in mate attraction may only become important at a later stage, while young

379 females rather use their energy for feeding and producing eggs. Due to the low sample sizes and

380 the low activity of males in that year, we did not find effects of condition within the class of old

381 females. More experiments are necessary to investigate whether females of different condition

382 but of the same age attract more males.

383 Our findings are inconsistent with the result from Chinta and Schulz (unpublished data; reviewed 384 in Schulz 2013) that the production of the sex pheromone (trimethyl (2R,3S)-methylcitrate) of $A$.

385 bruennichi increases until the fourth day after the final moult and then decreases. Since male

386 attraction by this sex pheromone follows a concentration-dependent matter (Chinta et al. 2010),

387 the middle-aged females of our study should have attracted more males than the old females. An

388 explanation for these inconsistencies could be that females close to oviposition attract males with 389 another semiochemical than trimethyl (2R, 3S)-methylcitrate. At least one other female specific 390 compound is known (Chinta et al. 2010). Whether or not increased mate attraction of old $A$. 391 bruennichi females is based on trimethyl (2R, 3S)-methylcitrate has to be resolved in further 392 studies.

393 The use of a different semiochemical shortly before oviposition could be based on a signal or cue 394 mechanism. Old females may actively enhance signalling with a further, perhaps more potent 395 pheromone, if the risk of laying unfertilized eggs outweighs the costs of attracting too many 396 males. Alternatively, females may passively emit oviposition cues or stress hormones, if the risk 397 of laying unfertilized eggs increased. Note that it is also adaptive for males to find virgin females 398 close to oviposition because the probablility might be lower, that females die before they 399 deposited their first egg sac (Rittschof 2011). 
400 Research on the praying mantis Pseudomantis albofimbriata (Barry 2010; Barry et al. 2010)

401 suggests that pheromone production could be directly linked to egg production and hence be

402 considered a cue rather than a signal. In a simultaneous choice test, male mantises displayed a

403 preference for females that carried more eggs, which correlates with body condition. Given a

404 choice between two females in equally poor condition, the males still picked the one with more

405 eggs in their ovaries (Barry 2010; Barry et al. 2010). This link between pheromone release and

406 fecundity could be mediated by juvenile hormone titers (Barry 2010). This explanation is

407 unlikely to apply to spiders with strong first male sperm precedence such as in $A$. bruennichi

408 (Austad 1982; Jones \& Parker 2008; Nessler et al. 2007; Uhl et al. 2014). Most females will mate

409 shortly after maturation and are no longer visited by males, most likely because mated females

410 stop producing pheromones (Chinta et al. 2010). Copulation with mated females promises low

411 fitness returns for males since the genital openings are blocked with genital parts of the

412 predecessor (Nessler et al. 2007) and unlike in mantises where a male constitutes a significant

413 nutritional benefit (Barry et al. 2008; Birkhead et al. 1988; Maxwell 2000), males of Argiope are

414 very small and provide only a small addition to the diet (Blamires 2011; Fromhage et al. 2003).

415 However, egg maturation is a continuous process and independent of mating. Hence, females

416 that mate shortly after maturation and are no longer interesting for males, still develop their eggs

417 at much the same speed as unmated females. To understand the mechanisms of dynamic

418 pheromone emission, we need studies that examine the underlying physiological processes.

419 Pheromone emission in spiders is poorly understood. The glands responsible for sex pheromone

420 secretion and the mechanism to attach them to the web silk are still unknown. Just a few studies

421 investigated if female spiders use both their body and their web for mate attraction and no study

422 compared the efficiency. For wide range-attraction, volatile pheromones rather than contact 
423 pheromones are used (Kasumovic \& Andrade 2004; Olive 1982; Riechert \& Singer 1995; Searcy

424 et al. 1999). Prouvost and colleagues (1999) found that in the house spider Tegenaria atrica, the

425 body cuticle and the web differ in some chemical compounds. Possibly, the importance of female

426 or web pheromones for mate attraction depends on the mobility of the spider species. In orb-web

427 spiders, females are sessile and mainly use volatile pheromones for mate attraction while web

428 silk might give males more specific information about the female upon contact. In wandering

429 spiders, silk, especially from draglines, may have a higher relevance for mate attraction and mate

430 search than the silk of orb-webs (discussed in Baruffaldi et al. 2010).

431 As far as we know, this is the first study that compared the efficiency of pheromones transmitted

432 by web silk and body in the field. Beyond that, our study complements the picture of

433 mechanisms of mate attraction in spiders. In most studies, age-dependent mate attraction was

434 explained by female receptivity (Klein et al. 2012; Papke et al. 2001; Roberts \& Uetz 2005). We

435 can exclude this explanation because $A$. bruennichi females are always receptive (Schneider et

436 al. 2006). We can show that beside moths (Umbers et al. 2015), female spiders adjust mate

437 attraction to the pressure of achieving a copulation. Future research should combine female age

438 and mating status to test when females stop signalling. A. bruennichi females are polyandrous

439 and benefit from attracting males after the first copulation.

440

441 Conclusion

442 We conclude that female signalling is a dynamic process and depends on the time spent

443 unfertilised. Female signalling seems to be strategic implying the presence of costs. We found

444 that the signal strength variation was adaptive and likely adjusted to the females' needs. 
446 Acknowledgements

447 We thank Tomma Dirks, Angelika Taebel-Hellwig, Gabriele Uhl, and Stefanie Zimmer for their 448 crucial support during the experimental planning, the collection of spiders, and the experimental

449 conduction in the field. Furthermore, we like to thank Jasmin Ruch and two anonymous

450 reviewers for helpful comments on the manuscript. 


\section{REFERENCES}

453 Andersson M. 1994. Sexual Selection. Princeton Univ Press.

454 Arakaki N, Wakamura S, and Yasuda T. 1996. Phoretic egg parasitoid, Telenomus euproctidis

455 (Hymenoptera: Scelionidae), uses sex pheromone of tussock moth Euproctis taiwana

456

457 (Lepidoptera: Lymantriidae) as a kairomone. Journal of Chemical Ecology 22:1079-

458

459

460

461

462

463

464

465

466

467

468

469

470

471

472

473

474 1085. 10.1007/BF02027946

Arnqvist G, and Nilsson T. 2000. The evolution of polyandry: multiple mating and female fitness in insects. Animal Behaviour 60:145-164. 10.1006/anbe.2000.1446

Arnqvist G, and Rowe L. 2005. Sexual conflict. Princton Univ Press.

Austad SN. 1982. First Male Sperm Priority in the Bowl and Doily Spider, Frontinella pyramitela (Walckenaer). Evolution 36:777. 10.2307/2407891

Barry KL. 2010. Influence of female nutritional status on mating dynamics in a sexually cannibalistic praying mantid. Animal Behaviour 80:405-411. 10.1016/j.anbehav.2010.05.024

Barry KL, Holwell GI, and Herberstein ME. 2008. Female praying mantids use sexual cannibalism as a foraging strategy to increase fecundity. Behavioral Ecology 19:710-715. 10.1093/beheco/arm 156

Barry KL, Holwell GI, and Herberstein ME. 2010. Multimodal mate assessment by male praying mantids in a sexually cannibalistic mating system. Animal Behaviour 79:1165-1172. 10.1016/j.anbehav.2010.02.025

Baruffaldi L, Costa FG, Rodriguez A, and Gonzalez A. 2010. Chemical Communication in Schizocosa malitiosa: Evidence of a Female Contact Sex Pheromone and Persistence in the Field. Journal of Chemical Ecology 36:759-767. 10.1007/s10886-010-9819-x 
475 Birkhead TR, Lee KE, and Young P. 1988. Sexual Cannibalism in the Praying Mantis Hierodula 476 membranacea. Behaviour 106:112-118. Doi 10.1163/156853988x00115

477

478

479

480

481

482

483

484

485

486

487

488

489

490

491

492

493

494

495

496

Blamires SJ. 2011. Nutritional implications for sexual cannibalism in a sexually dimorphic orb web spider. Austral Ecology 36:389-394. 10.1111/j.1442-9993.2010.02161.x

Bradbury JW, and Vehrencamp SL. 1998. Principles of animal communication.

Chinta SP, Goller S, Lux J, Funke S, Uhl G, and Schulz S. 2010. The sex pheromone of the wasp spider Argiope bruennichi. Angewandte Chemie-International Edition 49:2033-2036. 10.1002/anie.200906311

Endler JA. 1993. Some general comments on the evolution and design of animal communication systems. Philos Trans R Soc Lond B Biol Sci 340:215-225. 10.1098/rstb.1993.0060

Foellmer MW, and Fairbairn DJ. 2003. Spontaneous male death during copulation in an orbweaving spider. Proceedings of the Royal Society B Biological sciences 270 Suppl 2:S183-185. 10.1098/rsb1.2003.0042

Fromhage L, Uhl G, and Schneider JM. 2003. Fitness consequences of sexual cannibalism in female Argiope bruennichi. Behavioral Ecology and Sociobiology 55:60-64. $10.1007 / \mathrm{s} 00265-003-0656-6$

Gaskett AC. 2007. Spider sex pheromones: emission, reception, structures, and functions. Biological Reviews 82:26-48. 10.1111/j.1469-185X.2006.00002.x

Gerhardt HC. 1994. The Evolution of Vocalization in Frogs and Toads. Annual Review of Ecology and Systematics 25:293-324. DOI 10.1146/annurev.es.25.110194.001453

Greenfield MD. 1981. Moth Sex-Pheromones - an Evolutionary Perspective. Florida Entomologist 64:4-17. Doi 10.2307/3494597 
497 Harari AR, and Steinitz H. 2013. The evolution of female sex pheromones. Current Zoology

498

499

500

501

502

503

504

505

506

507

508

509

510

511

512

513

514

515

516

517

518 59:569-578.

Hector KL, and Nakagawa S. 2012. Quantitative analysis of compensatory and catch-up growth in diverse taxa. Journal of Animal Ecology 81:583-593. 10.1111/j.13652656.2011.01942.x

Herberstein ME, Schneider JM, and Elgar MA. 2002. Costs of courtship and mating in a sexually cannibalistic orb-web spider: female mating strategies and their consequences for males. Behavioral Ecology and Sociobiology 51:440-446. 10.1007/s00265-002-0460-8

Hoglund J, and Alatalo RV. 1995. Monographs in Behavior and Ecology: Leks.

Jerhot E, Stoltz JA, Andrade MC, and Schulz S. 2010. Acylated serine derivatives: a unique class of arthropod pheromones of the Australian redback spider, Latrodectus hasselti. Angewandte Chemie-International Edition 49:2037-2040. 10.1002/anie.200906312

Johnson JC, Trubl P, Blackmore V, and Miles L. 2011. Male black widows court well-fed females more than starved females: silken cues indicate sexual cannibalism risk. Animal Behaviour 82:383-390. 10.1016/j.anbehav.2011.05.018

Jones TC, and Parker PG. 2008. First male sperm precedence in multiply-mated females of the cooperative spider Anelosimus studiosus (Araneae, Theridiidae). Journal of Arachnology 36:527-532. Doi 10.1636/St06-63.1

Kaissling KE, and Priesner E. 1970. Olfactory Threshold of Silk Moths. Naturwissenschaften $57: 23-\&$

Kasumovic MM, and Andrade MCB. 2004. Discrimination of airborne pheromones by matesearching male western black widow spiders (Latrodectus hesperus): species- and 

Zoologie 82:1027-1034. 10.1139/Z04-081

521 Klein AL, Trillo MC, and Albo MJ. 2012. Sexual receptivity varies according to female age in a 522 Neotropical nuptial gift-giving spider. Journal of Arachnology 40:138-140.

523 Kokko H, and Wong BBM. 2007. What determines sex roles in mate searching? Evolution 61:1162-1175. 10.1111/j.1558-5646.2007.00090.x

Lubin YD. 1986. Courtship and Alternative Mating Tactics in a Social Spider. Journal of

527

528

529

530

531

532

533

534

535

536

537

538

539 Arachnology 14:239-257.

Magnhagen C. 1991. Predation Risk as a Cost of Reproduction. Trends in Ecology \& Evolution 6:183-185. Doi 10.1016/0169-5347(91)90210-O

Maxwell MR. 2000. Does a single meal affect female reproductive output in the sexually cannibalistic praying mantid Iris oratoria? Ecological Entomology 25:54-62. 10.1046/j.1365-2311.2000.00227.x

Nessler SH, Uhl G, and Schneider JM. 2007. Genital damage in the orb-web spider Argiope bruennichi (Araneae : Araneidae) increases paternity success. Behavioral Ecology 18:174-181. 10.1093/beheco/ar1074

Noldus L, Potting RPJ, and Barendregt HE. 1991. Moth sex pheromone adsorption to leaf surface: bridge in time for chemical spies. Physiological Entomology 16:329-344. 10.1111/j.1365-3032.1991.tb00571.x

Olive CW. 1982. Sex Pheromones in Two Orbweaving Spiders, (Aranaeae, Araneidae) - An Experimental Field Study. Journal of Arachnology 10:241-245. 
540 Papke MD, Riechert SE, and Schulz S. 2001. An airborne female pheromone associated with 541 male attraction and courtship in a desert spider. Animal Behaviour 61:877-886. 10.1006/anbe. 2000.1675

543 Perampaladas K, Stoltz JA, and Andrade MCB. 2008. Mated redback spider females re-advertise 544 receptivity months after mating. Ethology 114:589-598. 10.1111/j.14390310.2008.01513.x

546 Prouvost O, Trabalon M, Papke M, and Schulz S. 1999. Contact sex signals on Web and cuticle of Tegenaria atrica (Araneae, Agelenidae). Archives of Insect Biochemistry and Physiology 40:194-202. 10.1002/(sici)1520-6327(1999)40:4<194::aid-arch4>3.0.co;2-p

549

550

551

552

553

554

555

556

557

558

559

560

561

562

Rabaneda-Bueno R, Rodriguez-Girones MA, Aguado-de-la-Paz S, Fernandez-Montraveta C, De Mas E, Wise DH, and Moya-Larano J. 2008. Sexual Cannibalism: High Incidence in a Natural Population with Benefits to Females. PLoS ONE 3. 10.1371/journal.pone.0003484

Riechert SE, and Singer FD. 1995. Investigation of potential male mate choice in a monogamous spider. Animal Behaviour 49:715-723. 10.1016/0003-3472(95)90046-2

Rittschof CC. 2011. Mortality risk affects mating decisions in the spider Nephila clavipes. Behavioral Ecology 22:350-357. 10.1093/beheco/arq222

Roberts JA, and Uetz GW. 2005. Information content of female chemical signals in the wolf spider, Schizocosa ocreata: male discrimination of reproductive state and receptivity. Animal Behaviour 70:217-223. 10.1016/j.anbehav.2004.09.026

Robinson MH, and Robinson B. 1973. Ecology and behaviour of the giant wood spider Nephila maculata (Fabricius) in New Guinea. Smithsonian Contributions to Zoology No. 149:175. 
563 Roelofs WL. 1995. Chemistry of sex attraction. Proceedings of the National Academy of 564 Sciences 92:44-49.10.1073/pnas.92.1.44

565 Schneider JM, Fromhage L, and Uhl G. 2005. Extremely Short Copulations Do Not Affect 566 Hatching Success in Argiope bruennichi (Araneae, Araneidae). Journal of Arachnology 33:663-669. 10.1636/s03-32.1

568 Schneider JM, Gilberg S, Fromhage L, and Uhl G. 2006. Sexual conflict over copulation 569 duration in a cannibalistic spider. Animal Behaviour 71:781-788. 10.1016/j.anbehav.2005.05.012

571 Schneider JM, Zimmer SM, Gatz AL, and Sauerland K. 2016. Context- and State-Dependent Male Mate Choice in a Sexually Cannibalistic Spider. Ethology 122:257-266. DOI: 10.1111/eth.12466

574 Schulte-Hostedde AI, Zinner B, Millar JS, and Hickling GJ. 2005. Restitution of mass-size 575 residuals: Validating body condition indices. Ecology 86:155-163. Doi 10.1890/04-0232

576 Schulte KF, Uhl G, and Schneider JM. 2010. Mate choice in males with one-shot genitalia: 577 limited importance of female fecundity. Animal Behaviour 80:699-706. $578 \quad$ 10.1016/j.anbehav.2010.07.005

579 Schulz S. 2013. Spider Pheromones - a Structural Perspective. Journal of Chemical Ecology $580 \quad 39: 1-14.10 .1007 / \mathrm{s} 10886-012-0231-6$

581 Schulz S, Cardé RT, and Millar JG. 2004. Semiochemistry of spiders. Advances in Insect 582 Chemical Ecology:110-150. 10.1017/cbo9780511542664.005

583 Schulz S, and Toft S. 1993. Identification of a Sex Pheromone from a Spider. Science 260:1635584 1637. $10.1126 /$ science. 260.5114 .1635 
585 Searcy LE, Rypstra AL, and Persons MH. 1999. Airborne chemical communication in the wolf 586 spider Pardosa milvina. Journal of Chemical Ecology 25:2527-2533. Doi 10.1023/A:1020878225553

588

589

590

591

592

593

594

595

596

597

598

599

600

601

602

603

604

605

606

607

Steinbrecht RA. 1970. Morphometric Studies on Antenna of Silk Moth, Bombyx mori L. Number and Distribution of Olfactory Sensilla. Zeitschrift Fur Morphologie Der Tiere 68:93-126.

Stoltz JA, McNeil JN, and Andrade MCB. 2007. Males assess chemical signals to discriminate just-mated females from virgins in redback spiders. Animal Behaviour 74:1669-1674. 10.1016/j.anbehav.2007.03.011

Sweger AL, Marr M, Satteson A, Rypstra AL, and Persons MH. 2010. The effects of predation risk on female silk deposition and male response to predator-cued conspecifics in the wolf spider, Pardosa milvina (Araneae: Lycosidae). Journal of Arachnology 38:393-397. Doi 10.1636/Hi09-89.1

Thomas ML. 2011. Detection of female mating status using chemical signals and cues. Biological Reviews 86:1-14. 10.1111/j.1469-185X.2010.00130.x

Tillman JA, Seybold SJ, Jurenka RA, and Blomquist GJ. 1999. Insect pheromones - an overview of biosynthesis and endocrine regulation. Insect Biochemistry and Molecular Biology 29:481-514. 10.1016/s0965-1748(99)00016-8

Tuni C, and Berger-Tal R. 2012. Male preference and female cues: males assess female sexual maturity and mating status in a web-building spider. Behavioral Ecology 23:582-587. 10.1093/beheco/ars001

Uhl G, Kunz K, Vocking O, and Lipke E. 2014. A spider mating plug: origin and constraints of production. Biological Journal of the Linnean Society 113:345-354. 10.1111/bij.12359 
608 Uhl G, Zimmer SM, Renner D, and Schneider JM. 2015. Exploiting a moment of weakness:

609 male spiders escape sexual cannibalism by copulating with moulting females. Scientific

$610 \quad$ Reports 5. $10.1038 /$ srep 16928

611 Umbers KD, Symonds MR, and Kokko H. 2015. The mothematics of female pheromone

612 signaling: strategies for aging virgins. Am Nat 185:417-432. 10.1086/679614

613

614

615

616

617

618

619

620

621

622

623

624

625

626

627

628

629 van Ginneken VJT, and Maes GE. 2005. The European eel (Anguilla anguilla, Linnaeus), its Lifecycle, Evolution and Reproduction: A Literature Review. Reviews in Fish Biology and Fisheries 15:367-398. 10.1007/s11160-006-0005-8

Wall C, and Perry JN. 1987. Range of Action of Moth Sex-Attractant Sources. Entomologia Experimentalis Et Applicata 44:5-14.

Watson PJ. 1986. Transmission of a Female Sex-Pheromone Thwarted by Males in the Spider Linyphia litigiosa (Linyphiidae). Science 233:219-221. 10.1126/science.3726530

Welke KW, and Schneider JM. 2012. Sexual cannibalism benefits offspring survival. Animal Behaviour 83:201-207. 10.1016/j.anbehav.2011.10.027

Welke KW, Zimmer SM, and Schneider JM. 2012. Conditional monogyny: female quality predicts male faithfulness. Frontiers in Zoology 9. 10.1186/1742-9994-9-7

Williams GC. 1992. Natural selection: domains, levels and challenges.

Wyatt TD. 2014. Pheromones and Animal Behavior: Chemical Signals and Signatures, 2nd Edition.

Zegelman L, Hassner A, Mendel Z, and Dunkelblum E. 1993. Synthesis and Field Bioassay of the Israeli Pine Bast Scale, Matsucoccus josephi, Female Sex-Pheromone. Tetrahedron Letters 34:5641-5644. Doi 10.1016/S0040-4039(00)73904-9 
630 Zimmer SM, Welke KW, and Schneider JM. 2012. Determinants of Natural Mating Success in 631 the Cannibalistic Orb-Web Spider Argiope bruennichi. PLoS ONE 7. $632 \quad 10.1371 /$ journal.pone.0031389

633 Zuk M, and Kolluru GR. 1998. Exploitation of sexual signals by predators and parasitoids. 634 Quarterly Review of Biology 73:415-438. 10.1086/420412

635 


\section{FIGURES}

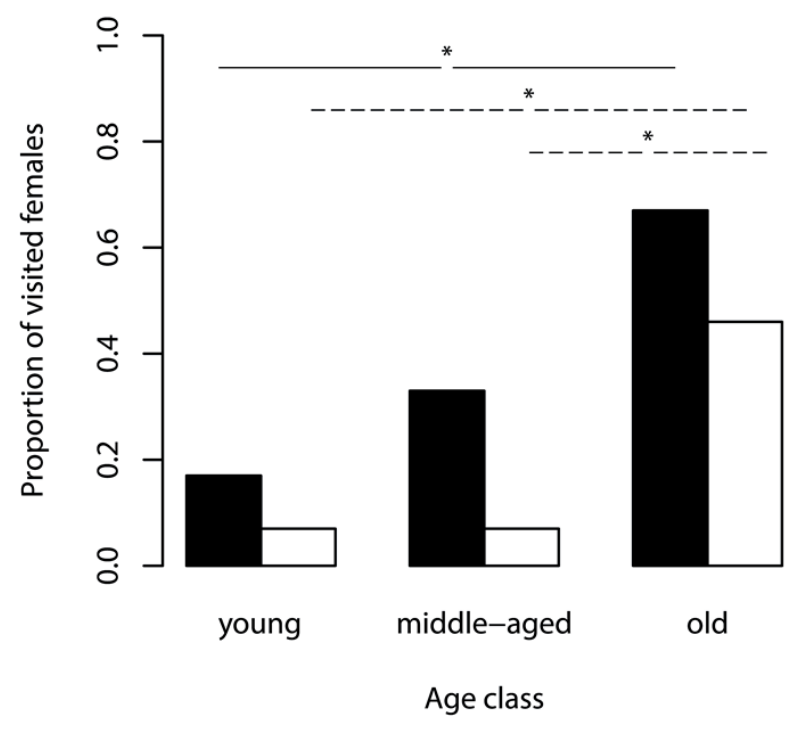

639

640 Figure 1: Proportion of visited females (black bars) and visited female webs (white bars)

641 depending on the female age. The solid lines (female body) and dashed lines (web silk) show

642 significant results between age classes. * = significant differences. 


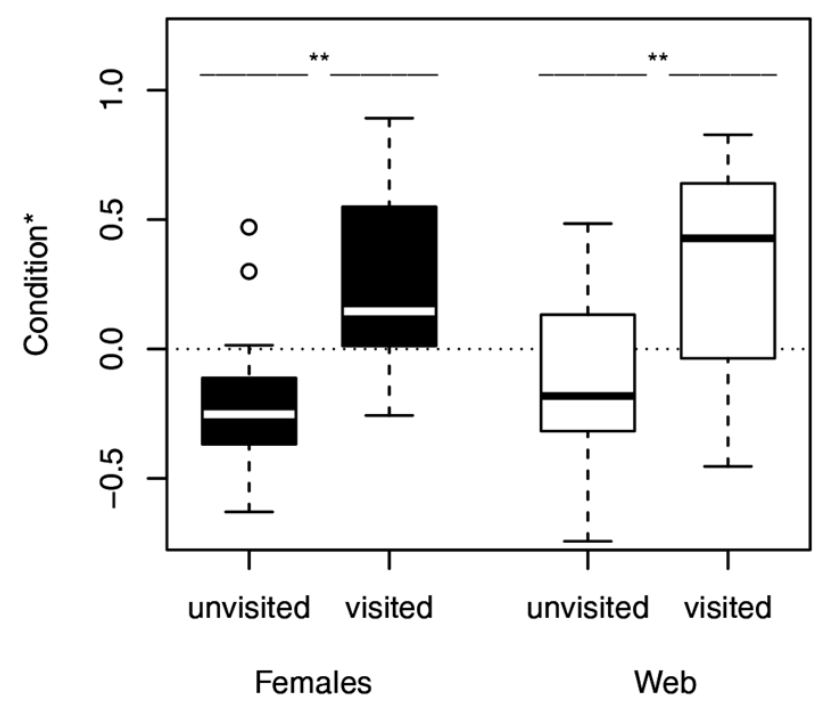

646 Figure 2: Condition of females separated into the occurrence of male visitation. Females and

647 their webs attracted more males, when they were in good condition. *The condition is shown as

648 the log-transformed residuals from the regression of weight and the size of the females. The

649 dotted line marks the zero line. 


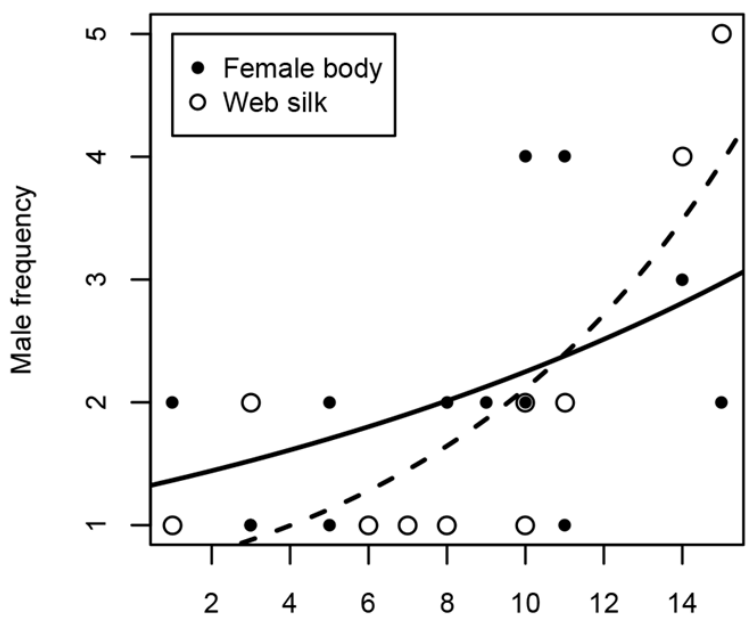

652 Post-mature age in days

653 Figure 3: Number of male visitors depending on female post-mature age. ${ }^{*}$ The lines show the 654 functional graphs for the "female body" data (solid line), the "web silk" data (dashed line). 